To the Editors:

\title{
Validating the Loop Mediated Isothermal Amplification (LAMP) technique to detect tuberculosis in a Sri Lankan laboratory setting
}

\author{
S U Perera ${ }^{1,2}$, V Navaratne ${ }^{1}$, A Nagahawatte ${ }^{3}$, J Perera ${ }^{4}$, C D Wijayarathna ${ }^{5}$, J Alvitigala ${ }^{6}$, \\ C L Goonasekara ${ }^{1}$
}

Ceylon Medical Journal 2018; 63: 40-42

DOI: http://doi.org/10.4038/cmj.v63i1.8625

\section{Introduction}

A critical obstacle to control tuberculosis (TB) caused by Mycobacterium tuberculosis (MTB) in the region is the lack of early and accurate diagnosis. The currently available methods for TB diagnosis, such as microscopy, culture, and PCR are either time consuming or less suitable for resource-limited rural settings. Previous studies have attempted to validate the Loop Mediated Isothermal Amplification (LAMP) assay as an alternative assay for TB diagnosis, as it is a simple and less time consuming technique that is more suitable for developing countries [1-4]. However, success has been limited due to a recurrent non-specific amplification of primers, leading to false positive results [5]. This paper describes the optimization of the LAMP assay, to avoid this false positive detection of MTB, and its efficacy as compared to the standard culture technique.

\section{Methodology}

Culture isolates prepared from clinical samples, which were collected from patients with suspected TB, at Department of Microbiology, University of Colombo, Sri Lanka and Welisara Chest Clinic, Sri Lanka were subjected to the LAMP assay. There were 46 mycobacteria culture positive samples. Based on conventional microscopy, culture results characteristics and biochemical tests, 31 of these cultures were pre-identified as Mycobacterium tuberculosis, and fifteen cultures as mycobacteria other than tuberculosis (MOTT). Colonies grown on Lowenstein Jensen (LJ) medium were lysed using heat sonication and the supernatant after the centrifugation of the lysate was subjected to the LAMP assay.
The LAMP reaction was carried out using Mycobacterium tuberculosis specific primers targeting the rimM gene sequence as previously described, with few modifications as follows; a. Varying amounts of SYBR dye; $1 \mu 1$ of $1 / 10$ diluted dye, $5 \mu 1$ of $1 / 10$ diluted dye and $1 \mu 1$ of undiluted dye, was added, $b$. Betaine at a concentration of $0.8 \mathrm{M}$ was also included in the reaction mixture, $\mathrm{c}$. The ZYBR green dye was placed on the inner-side of the cap of the microfuge tube containing the reaction mixture prior to amplification, and then the tube was spun after the amplification to mix the dye with the LAMP amplified products $[4,6]$.

\section{Results and Discussion}

The LAMP assay, according to the original protocol, with $1 \mu 1$ of $1 / 10$ diluted ZYBR green dye, resulted a green colour for the negative control, where there was no target DNA but simply distilled water [4]. When the negative reaction mixture after amplification was electrophoresed in $1 \%$ agarose gel, a low molecular weight band, corresponding to polymerized / dimerized primers could be identified, which appears to intercalate with ZYBR green giving a clear green colour even to the naked eye. This result indicated the false positive detection frequently observed with the LAMP assays.

To eliminate this primer dimerization, we varied the concentration of ZYBR green added and the primer concentration. When the LAMP amplification was detected by adding $1 \mu 1$ of undiluted ZYBR green dye instead of $1 \mu \mathrm{l}$ of $1 / 10$ diluted dye, in the presence of $0.8 \mathrm{M}$ betaine, the negative reaction appeared orange to the naked eye or under the UV light (Figure $1 \mathrm{~A} \& \mathrm{~B}$; assay 1).

${ }^{1}$ Faculty of Medicine, General Sir John Kotelawala Defence University, ${ }^{2}$ British College of Applied Sciences, Colombo, ${ }^{3}$ Department of Microbiology, Faculty of Medicine, University of Ruhuna, ${ }^{4}$ Department of Microbiology, Faculty of Medicine, University of Colombo, ${ }^{5}$ Department of Chemistry, Faculty of Science, University of Colombo, ${ }^{6}$ Central Chest Clinic and Hospital, Welisara, Sri Lanka.

Correspondence: CLG, e-mail: <charithalg@kdu.ac.lk>. Received 10 November 2017 and revised version accepted 05 January 2018

This is an open-access article distributed under the terms of the Creative Commons Attribution License, which permits unrestricted use, distribution, and reproduction in any medium, provided the original author and source are credited. 


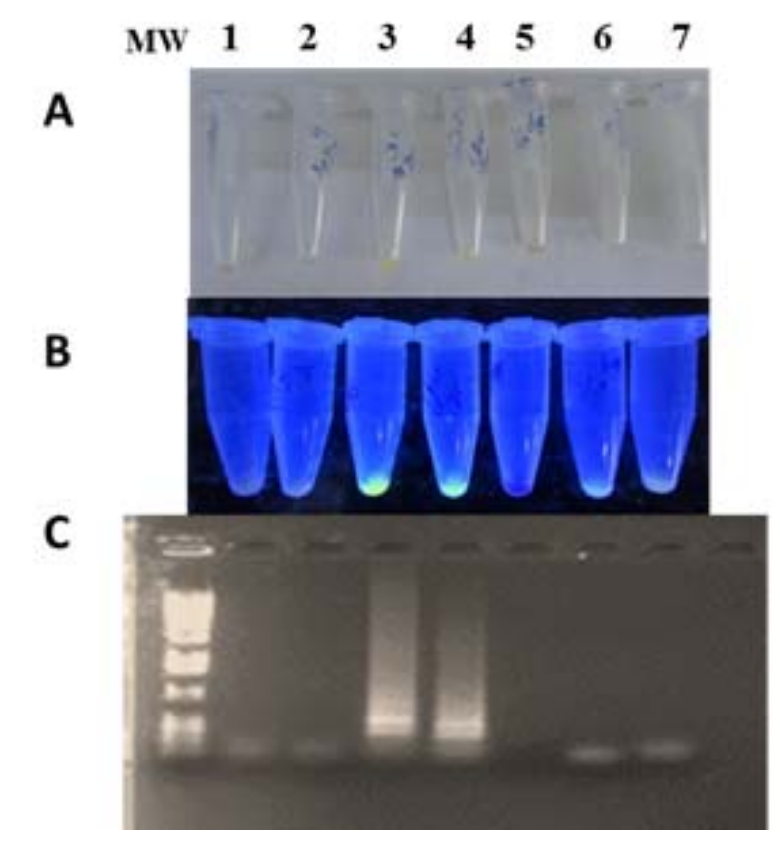

Figure 1. Optimization of the LAMP assay. LAMP amplified reactions mixtures were detected under A) normal light, B) UV light and C) in agarose gel electrophoresis.

When the ZYBR green concentration was reduced to either $1 \mu 1$ or $5 \mu 1$ of $1 / 10$ diluted dye, the green colour re-appeared in the negative reaction, but to a lesser extent with the latter (Figure $1 \mathrm{~A}$; assays $6 \& 7$ ). Figure $1 \mathrm{C}$ shows agarose gel electrophoresis of the same LAMP reactions, which shows that the intensity of the primer band decreased as the ZYBR green concentration is increased. Higher concentrations of the dye could possibly be reducing the primer dimerization / polymerization eliminating the false positive detection. Therefore, we conducted amplifications with a MOTT sample, the Mycobacterium tuberculosis positive control and a Mycobacterium tuberculosis sample, adding $1 \mu$ l of undiluted dye for detection, and the amplified products were electrophoresed, which are shown in lanes 2, 3 and 4 of the same Figure. The MOTT sample gave an orange colour just as the negative control, while the positive control and the Mycobacterium tuberculosis sample gave a green colour, which were clearly distinguishable with naked eye or under the UV light. Only the positive control or the Mycobacterium tuberculosis sample showed the presence of LAMP amplicons on the $1 \%$ agarose gel.

1. Negative control with $1 \mu 1$ of undiluted ZYBR green dye, 2. MOTT sample with $1 \mu 1$ of undiluted ZYBR green dye, 3. Positive control with $1 \mu 1$ of undiluted ZYBR green dye, 4. MTB sample with $1 \mu 1$ of undiluted ZYBR green dye, 5. Positive control with no primers, 6 . Negative control with $1 \mu 1$ of $1 / 10$ diluted dye, 7 . Negative control with $5 \mu 1$ of 1/10 diluted dye, MW; Molecular weight marker.
Based on the above optimization, we adapted the original protocol but with $1 \mu 1$ of undiluted ZYBR green dye for the detection of LAMP assay using naked eye or under the UV light. Thirty six samples were positive for LAMP assay while 10 were negative. Of the $31 \mathrm{Myco-}$ bacterium tuberculosis samples, all were also positive in the LAMP assays. Of the 15 MOTT samples, only 10 were identified as MOTT in the LAMP assay. Accordingly, the sensitivity of LAMP assay in detecting a Mycobacterium tuberculosis samples was $100 \%$. The specificity of identifying a non Mycobacterium tuberculosis samples as a MOTT was only $66.67 \%$ (Table 1). According to Keeler et al. a rapid test requiring less infrastructure facilities with a sensitivity and specificity of $85 \%$ and $97 \%$ can potentially avert $24 \%$ of world deaths due to TB [7]. Although we have not achieved such a high specificity with the LAMP assay in the current study under the tested conditions in local settings, and that it is low compared to other studies, the assay has room for improvement $[2,8$, 9]. For instance, when the reaction mixtures of the MOTT samples, after the LAMP assays, were run in $1 \%$ agarose gel (Figure 2), the reaction mixtures of the 5 false positives, showed the presence of some LAMP amplicons, rather than primer dimers, indicating a possibility of a cross contamination during the experi-mentation. Aerosol contamination by positive samples or positive amplicons has been reported as a common reason for getting false positives in the LAMP assay [10]. More careful handling of the samples could be practiced to minimize this nature of false positives, and simultaneously to increase the specificity of the assay.

\section{$\begin{array}{lllllll}1 & 2 & 3 & 4 & 5 & 6 & 7\end{array}$}

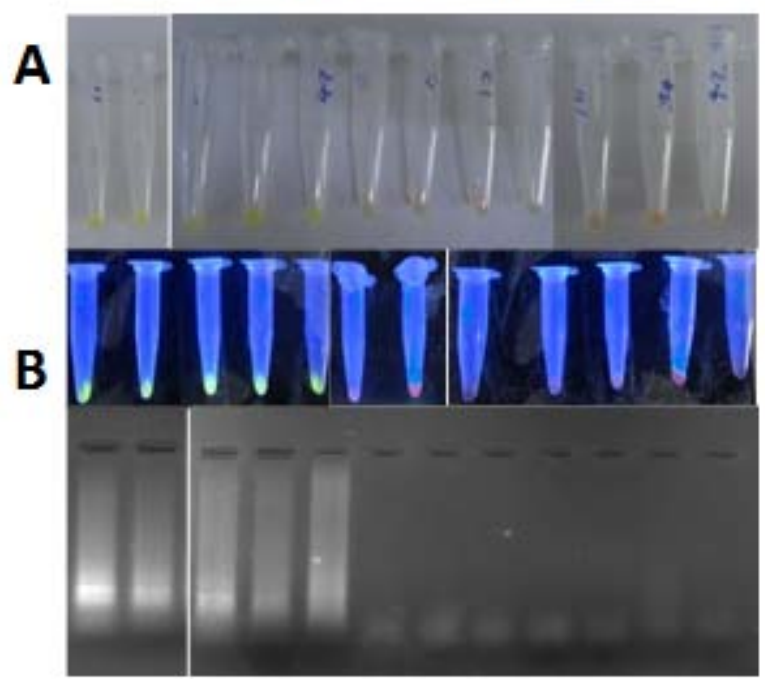

Figure 2. LAMP assays of MTB and MOTT samples. LAMP amplicons were detected under A) normal light, B) UV light and C) in agarose gel electrophoresis. 
Table 1. The sensitivity and specificity of LAMP assay in comparison to culture technique

\begin{tabular}{lcccc}
\hline & $\begin{array}{c}\text { Culture } \\
\text { technique } \\
\text { Positive }\end{array}$ & $\begin{array}{c}\text { Culture } \\
\text { technique } \\
\text { Negative }\end{array}$ & Sensitivity & Specificity \\
& & & \\
\hline $\begin{array}{l}\text { LAMP } \\
\text { assay }\end{array}$ & 31 & 5 & $100 \%$ & $66.67 \%$ \\
Positive & & & $88.78-$ & $38.38-$ \\
LAMP & 0 & 10 & $(\mathrm{CI} 95 \%)$ & $(\mathrm{CI} 95 \%)$ \\
assay & & & & \\
Negative & & & & \\
\hline
\end{tabular}

Overall, the LAMP assay shows to be a promising diagnostic tool for TB diagnosis. It is worth evaluating further to yield a reasonable level of sensitivity and specificity in detecting Mycobacterium tuberculosis in actual clinical samples.

\section{Conclusion}

The LAMP technique is a technically simple molecular based assay for the identification of Mycobacterium tuberculosis in culture isolates with high sensitivity and moderate specificity. It is worth validating the technique for the detection of Mycobacterium tuberculosis with acceptable efficacies in actual clinical samples.

$1-5 ; 5$ false positive MOTT samples-culture negative, LAMP positive; 6 - 12; 7 of the 10 true negative MOTT samples-both culture and LAMP negative. $1 \mu \mathrm{l}$ of undiluted ZYBR green dye are added for each reaction.

\section{Conflicts of interest}

There are no conflicts of interest.

\section{References}

1. Boehme CC, Nabeta P, Henostroza G et al. Operational feasibility of using loop-mediated isothermal amplification for diagnosis of pulmonary tuberculosis in microscopy centers of developing countries. J Clin Microbiol. 2007; 45: 1936-40.

2. Geojith G, Dhanasekaran S, Chandran SP, Kenneth J. Efficacy of loop mediated isothermal amplification (LAMP) assay for the laboratory identification of Mycobacterium tuberculosis isolates in a resource limited setting. J Microbiol Methods 2011; 84: 71-73.

3. Poon LL, Wong BW, Ma EH, et al. Sensitive and inexpensive molecular test for falciparum malaria: detecting Plasmodium falciparum DNA directly from heat-treated blood by loop-mediated isothermal amplification. Clin Chem. 2006; 52: 303-6.

4. Iwamoto T, Sonobe T, Hayashi K. Loop-mediated isothermal amplification for direct detection of Mycobacterium tuberculosis complex, M. avium, and M. intracellulare in sputum samples. J Clin Microbiol. 2003; 41: 2616-2622.

5. Senarath K, Usgodaarachchi R, Navaratne V, et al. Non Specific Amplification with the LAMP Technique in the Diagnosis of Tuberculosis in Sri Lankan Settings. J Tubercu Res. 2014; 2: 168-72.

6. Nagdev KJ, Kashyap RS, Parida MM, et al. Loopmediated isothermal amplification for rapid and reliable diagnosis of tuberculous meningitis. J Clin Microbiol. 2011; 49; 1861-5.

7. Keeler E, Perkins M, Small P, et al. Reducing the global burden of tuberculosis: the contribution of improved diagostics. Nature 2006; 444: 49-57.

8. Nliwasa M, MacPherson P, Chisala P, et al. The Sensitivity and Specificity of Loop-Mediated Isothermal Amplification (LAMP) Assay for Tuberculosis Diagnosis in Adults with Chronic Cough in Malawi. PLOS ONE 2016; 11: e0155101.

9. Kaewphinit T, Arunrut N, Kiatpathomchai W, Santiwatanakul S, Jaratsing P, Chansiri K. Detection of Mycobacterium tuberculosis by Using Loop-Mediated Isothermal Amplification Combined with a Lateral Flow Dipstick in Clinical Samples. BioMed Res Inter. 2013; 2013: 6 .

10. Hu S, Li M, Zhong L, Lu S, Liu Z, Pu J, Wen J, Huang X, Development of reverse-transcription loop-mediated isothermal amplification assay for rapid detection and differentiation of dengue virus serotypes 1-4. BMC Microbiol. 2015; 15: 265. 